\title{
Carrier-capture-assisted optoelectronics based on van der Waals materials to imitate medicine-acting metaplasticity
}

\author{
Qianfan Nie ${ }^{1,2,9}$, Caifang Gao ${ }^{1,9}$, Feng-Shou Yang ${ }^{3,4}$, Ko-Chun Lee ${ }^{3}$, Che-Yi Lin ${ }^{3}$, Xiang Wang ${ }^{1}$, Ching-Hwa Ho $\mathbb{E}^{5}$, Chen-Hsin Lien ${ }^{4}$, \\ Shu-Ping Lin $\mathbb{1}^{6,7}$, Mengjiao $\mathrm{Li}^{3,4 凶}$, Yen-Fu Lin ${ }^{3,7,8 凶}$, Wenwu Li $\mathbb{C}^{1,2 凶}$, Zhigao $\mathrm{Hu}^{1,2}$ and Junhao Chu ${ }^{1,2}$
}

Recently, researchers have focused on optoelectronics based on two-dimensional van der Waals materials to realize multifunctional memory and neuron applications. Layered indium selenide (InSe) semiconductors satisfy various requirements as photosensitive channel materials, and enable the realization of intriguing optoelectronic applications. Herein, we demonstrate InSe photonic devices with different trends of output currents rooted in the carrier capture/release events under various gate voltages. Furthermore, we reported an increasing/flattening/decreasing synaptic weight change index $\left(\Delta W_{n}\right)$ via a modulated gate electric field, which we use to imitate medicine-acting metaplasticity with effective/stable/ineffective features analogous to the synaptic weight change in the nervous system of the human brain. Finally, we take advantage of the low-frequency noise (LFN) measurements and the energy-band explanation to verify the rationality of carrier capture-assisted optoelectronics applied to neural simulation at the device level. Utilizing optoelectronics to simulate essential biomedical neurobehaviors, we experimentally demonstrate the feasibility and meaningfulness of combining electronic engineering with biomedical neurology.

npj 2D Materials and Applications (2021)5:60; https://doi.org/10.1038/s41699-021-00241-0

\section{INTRODUCTION}

The brain is the main intelligent organ. It has exclusive architecture that facilitates and executes highly energy efficient short-term computation, long-term learning, and memorization using the network of synapses in the neural system for data processing. The development of the next generation of humanoid robots and electronics is inseparably connected to the innovative concept of emulation and reproduction of biological systems and artificial synapse ${ }^{1,2}$. Recently, atomically thin van der Waals materials with characteristic internal and interfacial structures, large area development, and good charge transport behavior have attracted substantial interest in the field of neuromorphic engineering. The materials have enabled the development of synaptic devices, including resistive memory, sensors, and fieldeffect transistors (FETs) with various operating mechanisms such as ferroelectric or charge-trapping layer, electrochemical memory, and optoelectronic memory ${ }^{2-6}$. However, there is a common but inevitable problem in silicon substrate-based 2D electronic devices, numerous defects on the surface of the $\mathrm{SiO}_{2}$ originating from Si-O dangling bonds, which normally degrade either the electrical or optical performance of the device ${ }^{7-11}$. Therefore, modulating the external applied electric field to effectively control the carrier capture mechanism would be a major breakthrough in the development of artificial synaptic system ${ }^{12,13}$.

Synapses with functions of short-term plasticity (STP) and longterm plasticity (LTP), as the basic units of the human brain, and work in each brain circuit such as the reward pathway, which is the core pathway affected by drugs ${ }^{14-21}$. Synaptic plasticity is defined as the change in weight of the connections between neurons $\left(\Delta W_{n}\right.$ : the ratio between the postsynaptic responses triggered by the $\mathrm{n}^{\text {th }}$ and $1^{\text {st }}$ input spikes) $)^{22}$, and is analogous to long lasting of drug reward learning evoked by repeated drug stimulus. It represents three types of excitatory, flat, and inhibitory states through transmission of chemical media between the pre and post synapse. Likewise, in the drug reward pathway of the human brain, the $\Delta W_{n}$ index, which is increasing, flattening, and decreasing, can be used to characterize the three effects $21,23,24$, that is, effective, stable, and ineffective, when the neuronal networks are triggered by adequate, low and ultralow drug doses, respectively. At present, there is a great deal of research into the development of solid-state neuromorphic electronics that can simulate synaptic plasticity behaviors. However, the reported result about the $\Delta W_{n}$ index only exhibits a monotonous increase as either the stimuli frequency or pulse number increase $\mathrm{e}^{25-29}$. This cannot fully conform with the tunable increasing/flattening/ decreasing synaptic responses correlated with the "same" frequency or presynaptic spike number under different amplitude repeated stimulations in the neural circuits of the human brain. Different trends in the $\Delta W_{n}$ index have also been done but necessarily under "different" electrostatic pulses or optical pulses with different wavelength ${ }^{16,30-33}$. Based on the aforementioned mechanism, to effectively mimic the biological synapses at device level, the device needs to strongly depend on the polarity of the applied electric fields and possess the advantage of low power consumption. Advance of optically and electrically stimulated synaptic devices based on two-dimensional van der Waals layered materials $^{2,6,33}$ has been regarded as a thrilling progress in neuromorphic applications.

\footnotetext{
${ }^{1}$ Engineering Research Center for Nanophotonics \& Advanced Instrument (MOE), Technical Center for Multifunctional Magneto-Optical Spectroscopy (Shanghai), School of Physics and Electronic Science, East China Normal University, Shanghai, China. ${ }^{2}$ Institute for Optoelectronics, Shanghai Institute of Intelligent Electronics and Systems, Fudan University, Shanghai, China. ${ }^{3}$ Department of Physics, National Chung Hsing University, Taichung, Taiwan. ${ }^{4}$ Department of Electrical Engineering \& Institute of Electronic Engineering, National Tsing Hua University, Hsinchu, Taiwan. ${ }^{5}$ Graduate Institute of Applied Science and Technology, National Taiwan University of Science and Technology, Taipei, Taiwan. ${ }^{6}$ Graduate Institute of Biomedical Engineering, National Chung Hsing University, Taichung, Taiwan. ${ }^{7} \mathrm{i}-$ Center for Advanced Science and Technology (i-CAST), National Chung Hsing University, Taichung, Taiwan. ${ }^{8}$ Institute of Nanoscience, National Chung Hsing University, Taichung, Taiwan. ${ }^{9}$ These authors contributed equally: Qianfan Nie, Caifang Gao. ${ }^{\bowtie}$ email: mengjiaoli@email.nchu.edu.tw; yenfulin@nchu.edu.tw; wwli@ee.ecnu.edu.cn
} 
The present study demonstrated carrier capture-assisted optoelectronics, based on a two-dimensional van der Waals material indium selenide (InSe), comprising $\mathrm{Si} / \mathrm{SiO}_{2} / \mathrm{lnSe} / \mathrm{ln}$ layers and Au source-drain electrodes. It is proven that our layered InSe devices can imitate medicine-acting metaplasticity with three types of responses (effective/stable/ineffective) under various drug dosages. InSe, a III-VI group material with a low effective electron mass, excellent intrinsic charge transport characteristics, and ultrasensitive optical absorption, exhibits high on-off current ratio, carrier mobility, and excellent photoresponse compared with the pioneering works on other van der Waals semiconductorbased electronic devices ${ }^{6,34-38}$. The traps on the $\mathrm{SiO}_{2}$ surface that degrade device performances provide an opportunity to exploring carrier capture/release events between the InSe semiconductor and the $\mathrm{SiO}_{2}$ substrate by controlling electric field and applying optical pulses ${ }^{7,8,39-41}$. Therefore, we achieved a strong gatedependent persistent photocurrent and an increasing/flattening/ decreasing $\Delta W_{n}$ index, to successfully mimic the significant synaptic plasticity and drug response by positive/zero/negative correlations with "same" frequency and number of drug stimuli. Finally, the underlying mechanisms in terms of carrier capturing/ releasing characteristics were comprehensively illustrated and proved in detail by energy band and low-frequency noise (LFN) analysis ${ }^{34,42,43}$. In our work, we developed a drug dosage modulator utilizing InSe optoelectronics, which represents a significant step toward achieving the next generation of nanoelectronic devices based on artificial intelligence.

\section{RESULTS AND DISCUSSION}

\section{Device characterization and electrical/optical performances}

Since InSe possesses innate ultrahigh mobility and hypersensitivity to light, we fabricated optoelectronic devices based on layered InSe flakes to perform electrical measurements and probe exotic applications. Furthermore, the band gap of layered InSe transforms from direct to indirect as the material changes from bulk to a monolayer, which covers an extensive wavelength range from near-infrared to visible light ${ }^{44}$. Such advantages make InSe a desirable candidate for optoelectronic devices. What is more, as a two-dimensional material, InSe has the characteristic of low power consumption, which is further conducive to its application in the neuromorphic system. Figure 1a illustrates a schematic sketch of the back-gate InSe-based FET, and the optical image is shown in Fig. 1b. For stability and charge transport enhancement, an In layer was deposited on the top surface of the layered InSe semiconductor ${ }^{34}$. The thickness of the as-exfoliated and selected InSe flake, which was crudely determined by optical microscope with the help of color contrast and precisely measured by atomic force microscopy (AFM), was $\sim 11.6 \mathrm{~nm}$ (see Fig. 1c and Supplementary Fig. 1) for a better channel performance. To further understand the characteristic of the layered InSe, Raman spectrum was obtained by a $532 \mathrm{~nm}$ laser excitation (Fig. 1d). The characteristic peaks at $\sim 115, \sim 178, \sim 199$, and $\sim 228 \mathrm{~cm}^{-1}$ corresponding to vibration mode of $A_{1}^{\prime}\left(\Gamma_{1}^{2}\right), E^{\prime}\left(\Gamma_{1}^{2}\right)-\mathrm{TO}$, $A_{2}^{\prime \prime}\left(\Gamma_{1}^{1}\right)$-LO, and $A_{1}^{\prime}\left(\Gamma_{1}^{3}\right)$, respectively, were highly consistent with previous study ${ }^{45}$. Subsequently, we carried out photoelectric measurements to investigate the properties of the proposed design. The linear output characteristics $\left(I_{\mathrm{ds}}-V_{\mathrm{ds}}\right)$ of the InSe FETs under various gate voltages $\left(V_{\mathrm{bg}}\right)$, in Fig. $1 \mathrm{e}$, indicate ohmic contact between the metal electrodes and the InSe semiconductor capped with the In layer ${ }^{34}$. The transfer characteristics $\left(I_{\mathrm{ds}}-\right.$ $\left.V_{\mathrm{bg}}\right)$ at various illumination intensities of a 405-nm-wavelength laser, swept from -80 to $80 \mathrm{~V}$ at $V_{\mathrm{ds}}=0.5 \mathrm{~V}$, exhibited a high on/ off ratio of $10^{7}$ and a tremendous current density of $5.58 \times 10^{-5} \mathrm{~A}$ $\mu \mathrm{m}^{-1}$ in the dark under a relatively low drain-source voltage $\left(V_{\mathrm{ds}}\right)$, as shown in Fig. If. Moreover, as the light power density varied from 0 to $0.245 \mathrm{~W} \mathrm{~cm}^{-2}$, there was a distinct negative shift in the threshold voltage $\left(V_{\mathrm{th}}\right)$ owing to an increase in the concentration of photon-generated carriers ${ }^{36}$. Note that the photoelectrical characterizations were conducted in the dark to reduce fluctuations triggered by environmental disturbance. To quantitatively analyze the dependence of the photoresponse on the illumination intensity, the photocurrent $\left(I_{\text {ph }}=I_{\text {light }}-I_{\text {dark }}\right)$ was obtained at different values of $V_{\mathrm{bg}}$, with a drain-source voltage of $0.5 \mathrm{~V}$, as a function of the illumination power density (see Supplementary Fig. 2a). Figure $1 \mathrm{~g}$ illustrates the power law of $I_{\mathrm{ph}} \propto p^{a}$, where $P$ and $a$ represent the laser power density and the exponent factor, respectively. The correlation between $I_{\text {ph }}$ and $P$ was super linear $(a>1)$ at negative $V_{\text {bg }}$ value and sublinear $(a<1)$ at positive $V_{\mathrm{bg}}$ value. The results strongly hinted that there were trap states at the interface between the semiconductor and the dielectric layer, which can capture and release carriers and thereby influence charge transport ${ }^{46,47}$. Responsivity and detectivity are two other critical characteristics of the performance of optoelectronic InSe devices. Responsivity $(R)$ is defined as the photocurrent $\left(I_{\mathrm{ph}}\right)$ divided by the laser power $(P)$ and the illumination area of the device $^{48}(S)$ :

$R=I_{\mathrm{ph}} / P \cdot S$,

and is highly dependent on the $V_{\text {bg }}$ value, as shown in Fig. $1 \mathrm{~h}$. As the $V_{\text {bg }}$ increased from $-80 \mathrm{~V}$ to $80 \mathrm{~V}$, the variation in the responsivity with power density changed from ascent to descent. Supplementary Fig. $2 \mathrm{~b}$ presents the extracted $I_{\mathrm{ph}}$ and $R$ values at a fixed $V_{\mathrm{bg}}=0 \mathrm{~V}$, which exhibits an inverse trend with an incremental illumination intensity, in good agreement with recent research ${ }^{36}$. In Supplementary Fig. 2c, detectivity $\left(D^{*}\right)$, reflecting the sensitivity of a photoelectric InSe FET, was estimated as $^{48}$

$D^{*}=R \cdot \sqrt{S / 2 q l_{\text {dark }}}$,

where $q$ is the elementary charge, and revealed great uniformity with previous reports on InSe phototransistors ${ }^{36,49}$. It should be noted that these estimated optoelectronic parameters at a higher $V_{\mathrm{bg}}$ were relatively smaller, which was attributed to the small photocurrent variation under the device-saturation regime. However, all of these $I_{\mathrm{ph}}, R$, and $D^{*}$ values indicated the tunability of photoresponses, suggestively rooting in carrier-assisted InSe optoelectronics by adjusting the gate electric field.

\section{Mechanism of gate-modulated synaptic response}

To obtain a better understanding of the photoelectrical properties of InSe devices, we examined the performances under various gate voltages, $V_{\mathrm{bg}}$. As shown in Fig. $2 \mathrm{a}$, we plotted the temporal responses of an InSe-based phototransistor under the following conditions: $405-\mathrm{nm}$ wavelength and $0.245-\mathrm{W} \mathrm{cm}^{-2}$ power density, and $V_{\text {bg }}$ values of $-80,-40,0,40$, and $80 \mathrm{~V}$. Notably, the current decayed slower at lower $V_{\mathrm{bg}}$ values after the light was turned off and generated a persistent current at negative gate bias and $a$ very fast decay time at positive $V_{\text {bg }}$. Hypothetically, strong gatemodulated photoresponses benefit from significant carrier capture/release events at the interface between the InSe semiconductor and the $\mathrm{SiO}_{2}$ substrate. Further proof of this was provided by inserting another layered material comprising hexagonal boron nitride (h-BN) with free-dangling bonds into the InSe/SiO interface (see Supplementary Fig. 3). Obviously, InSe phototransistors based on an $\mathrm{h}-\mathrm{BN} / \mathrm{SiO}_{2}$ substrate have markedly a shorter relaxation time than phototransistors with $\mathrm{SiO}_{2}$ alone as the substrate ${ }^{50,51}$. It is noted that the dangling $\mathrm{Si}-\mathrm{O}$ bonds at the substrate surface play a crucial role in our carrier capture-assisted process. The dangling $\mathrm{Si}-\mathrm{O}$ bonds allow gate electric field to regulate the Fermi level on the surface of the InSe semiconductor, which facilitate carriers to be captured from the InSe channel to the $\mathrm{SiO}_{2}$ surface under positive $V_{\mathrm{bg}}$ and be released back under negative $V_{\mathrm{bg}}$. The phenomenon of channel conductance change caused by carrier release/capture processes, in line with the 


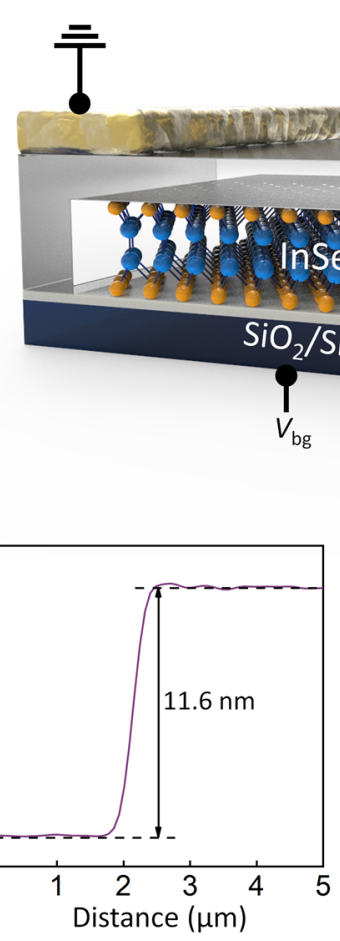

f

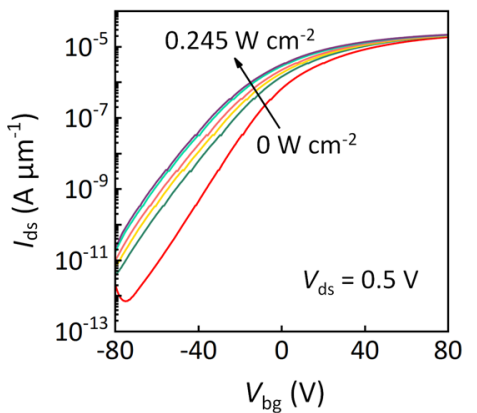

d

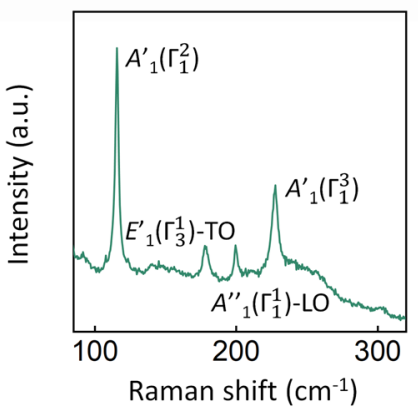

g

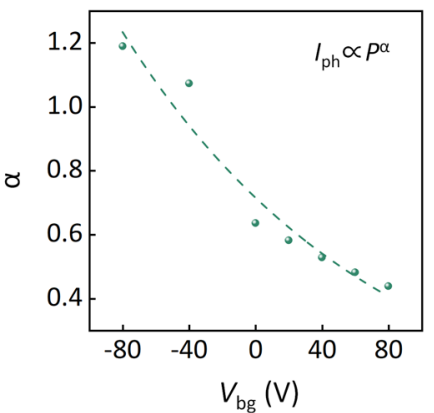

b

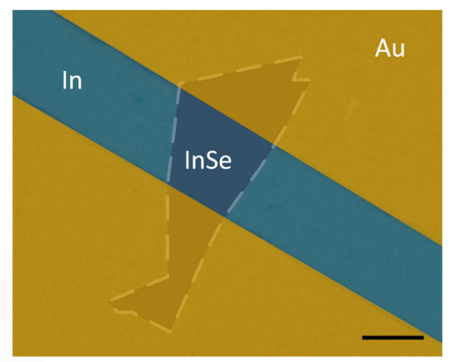

e

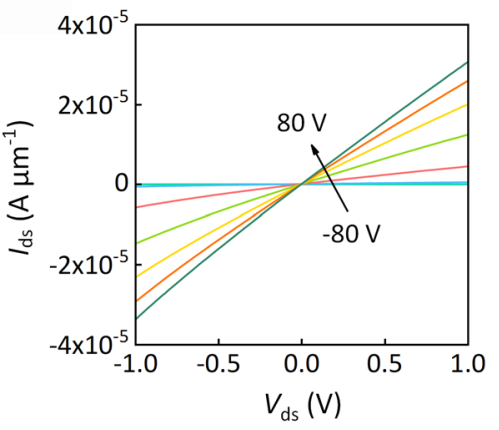

h

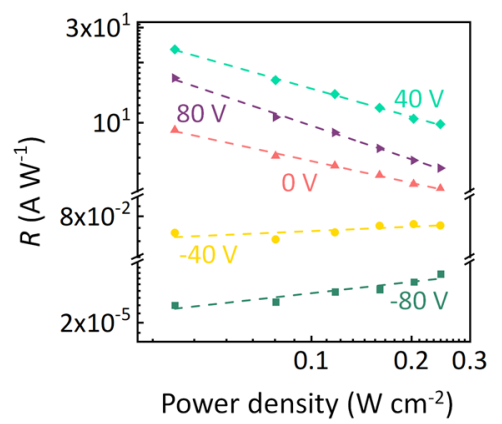

Fig. 1 Characterization, electrical and optical properties of multilayered InSe device. a Schematic sketch of the back-gate InSe-based device. b Optical image of the multilayered InSe device. Scale bar: $10 \mu \mathrm{m}$. c Height profile of the layered InSe channel. d Raman spectrum of the InSe flake with an excitation wavelength of $532 \mathrm{~nm}$. e Output characteristics of the InSe device tested in the dark at various $V_{\mathrm{bg}} . \mathbf{f}$ Transfer characteristics at different illumination intensities of a $405-\mathrm{nm}$ laser at $V_{\mathrm{ds}}=0.5 \mathrm{~V}$. $\mathbf{g}$ The extracted $a$ value as a function of $V_{\mathrm{bg}}$. $\mathbf{h}$ Responsivity $(R)$ of the InSe device as a function of the laser power density under diverse $V_{\mathrm{bg}}$ with $V_{\mathrm{ds}}=0.5 \mathrm{~V}$.

release/transport of neurotransmitters in the synapse ${ }^{16}$, provides the support for imitating essential synaptic plasticity behaviors including drug dosage reward-related metaplasticity. As presented in Fig. 2a, we applied a series of light pulses with the same laser wavelength, power density, and pulse width to mimic the action of a patient taking the same kind of drug, wherein the various gate electric fields served as different drug dosages. That is to say that positive/zero/negative bias represented ultralow/low/adequate drug dosages, respectively. In addition, the increasing optical spike frequency/pulse numbers are analogous to the frequency/ number of the drug taking, and the output device current can be taken as both a reward-related postsynaptic current (PSC) and a reward pathway response after drug stimulations or therapy. In the human brain, the reward pathway is an indispensable neural circuit, in which drugs mainly act through controlling the transmission of dopamine and other neurotransmitters ${ }^{24}$. When a drug stimulates and activates the reward pathway in patient's brain, the appropriate neurotransmitters are released from the presynaptic membrane to the synaptic space. They work by binding to receptors on the postsynaptic membrane, and are transported back to the pre-neurons with the aid of special transporters, after which the reward pathway provides positive feedback such as a feeling of wellbeing to patients. Namely, a beneficial medicine-acting reaction can be achieved and the health of the patient will get better and better and eventually reach saturation to recovery after repeated medication in adequate dosage. Whereas, a low or ultralow drug dosage stimulates the release of low levels of neurotransmitters and few changes in the postsynaptic membrane response ${ }^{17}$. The consequence for the human brain is that different drug doses (adequate/low/ultralow) give rise to three kinds of medicine-acting metaplasticity: effective, stable, and ineffective drug reactions. The results suggest that our proposed InSe optoelectronics have the potential to imitate medicine-acting metaplasticity by virtue of the operating gate electric field, as discussed in detail later. 


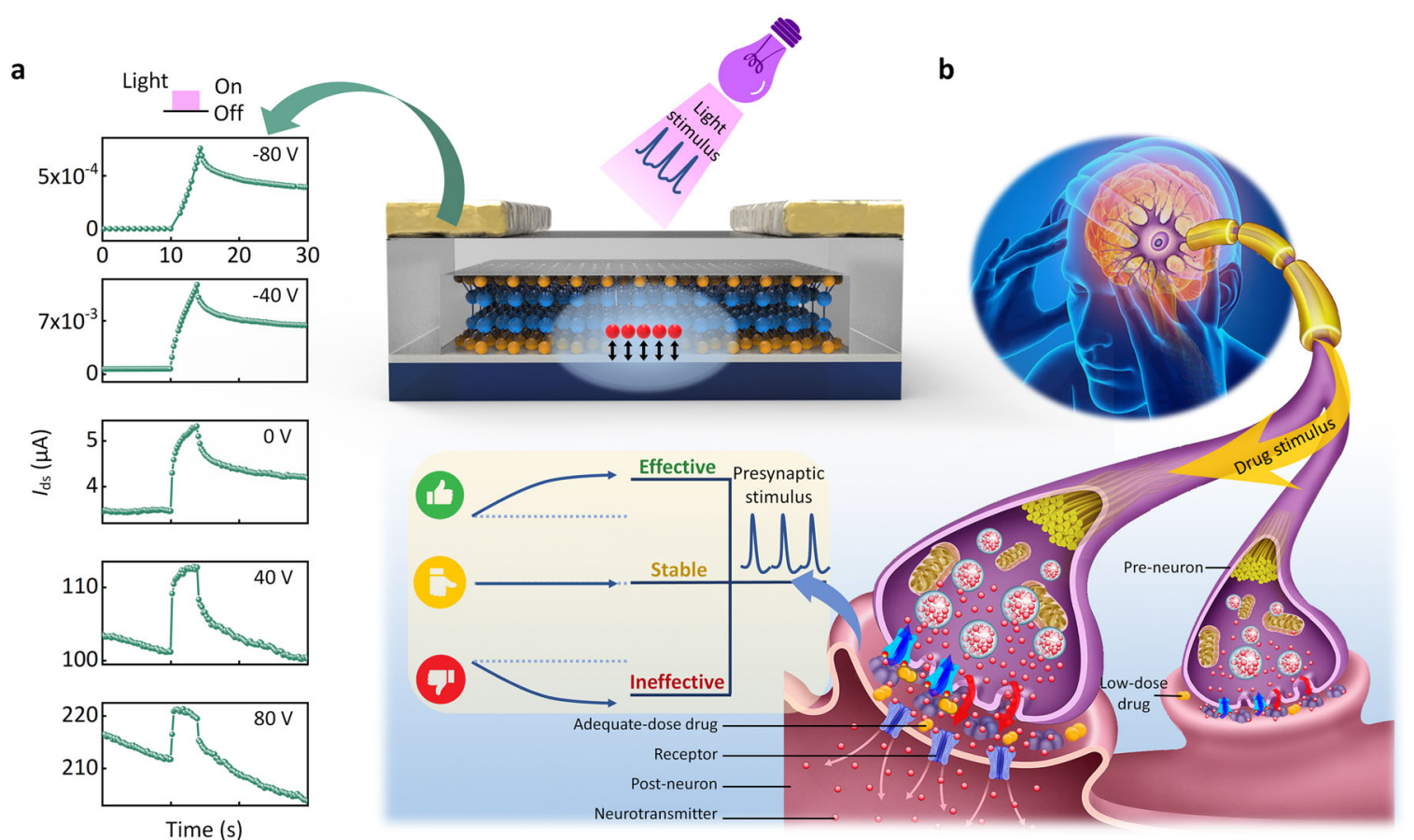

Fig. 2 Gate voltage-dependent electrical property and schematic diagram of the synaptic response mechanism. a Drain-source currents $\left(I_{\mathrm{ds}}\right)$ of the InSe synaptic device at gate voltages of $-80,-40,0,40$, and $80 \mathrm{~V}$ and drain-source voltage of $0.5 \mathrm{~V}$. b Schematic diagram illustrating the synaptic response mechanism and three outcomes of drug action in the nervous system. Used with permission from Guangzhou Inmagine Information Technology Co., Ltd, China, and Licensor (Seal) Shanghai Tuchong Network Technology Co., Ltd, China.

\section{Medicine-acting synaptic plasticity emulation}

In view of the aforementioned research and the discussion of the gate voltage regulation of the photoresponse and the neuroregulatory mechanism of drug dose in patients, we further verified that the artificial InSe-based devices are capable of imitating synaptic behavior. Synapses, as a bridge between neurons, transmit information by releasing and receiving neurotransmitters, which stimulates postsynaptic neurons to generate action potentials, leading to metaplasticity. Synaptic plasticity (i.e., STP and LTP) characterizes changes in the strength of synaptic weight aroused by memorization events ${ }^{16,18}$ and is manifested in the ability of the brain to learn and memorize the drug efficacy. To emulate experimentally STP and LTP behaviors in the InSe device, we adopted a 405-nm-wavelength laser and measured under $V_{\mathrm{bg}}$ $=-40 \mathrm{~V}$ and $V_{\mathrm{ds}}=0.5 \mathrm{~V}$. Figure $3 \mathrm{a}, \mathrm{b}$ records the PSCs in the InSe photoelectronic device illuminated by a series of laser pulses during an exposure cycle of $20 \mathrm{~s}$ (serving as presynaptic spikes or drug stimuli) at frequencies of 0.2 and $1 \mathrm{~Hz}$, respectively. In our measurements, the laser power density was kept constant as $0.245 \mathrm{~W} \mathrm{~cm}^{-2}$ and the pulse width was $500 \mathrm{~ms}$. In Fig. 3a, at the low frequency, it can be seen that the PSC decreased abruptly from $7.9 \mathrm{nA}$ to a steady state value of $4.9 \mathrm{nA}$ after the presynaptic spikes, causing STP behavior. Note that although STP means that the PSC return toward the initial value after the termination of stimulus within a short time, it eventually converges to a certain value that may not accord with the initial one as well. In order to make the effect of presynaptic stimulus more significant, we ultimately adopted above result. By contrast, when the InSe synaptic device was stimulated by high-frequency presynaptic spikes, the amplitude of the PSC slowly recovered stability and maintained a higher current value of $12.3 \mathrm{nA}$, shown in Fig. $3 \mathrm{~b}$. Therefore, we concluded that the relatively high-frequency drug stimulus exaggerated the changes in the synaptic weight, giving rise to an LTP behavior and a better persistent treatment effect. Figure $3 c$ exhibits the channel current of the InSe synaptic device and the calculated weight variation of the PSC $\left(\Delta W_{1}\right.$ : the ratio of
PSC change induced by the presynaptic spike to the initial current level) under various pulse widths at the same power density of $0.245 \mathrm{~W} \mathrm{~cm}^{-2}$. When the pulse width was modified from 0.05 to $10 \mathrm{~s}$, the strength of the PSC and the value of $\Delta W_{1}$ enhanced gradually. We observed similar results following an increase in the illumination intensity at a fixed stimulation duration of $500 \mathrm{~ms}$, as shown in Fig. $3 \mathrm{~d}$. Therefore, the amplitude of persistent $I_{\mathrm{ph}}$ is able to be effectively tuned by varying the width and power of the laser pulse because of various channel conductance in InSe optoelectronics, which can mimic the synaptic plasticity events that occur during treatment with different types of medication.

Paired-pulse facilitation (PPF) is another crucial manifestation of STP. It relates to the recognition and decoding of temporal information about the drug stimulus and represents the synaptic responses which can be strengthened by the effects of two successive presynaptic spikes ${ }^{1,52,53}$. PPF was emulated in the InSe device to explore dynamic synaptic plasticity as well. Two identical laser pulses (power intensity $0.245 \mathrm{~W} \mathrm{~cm}^{-2}$, exposure duration $500 \mathrm{~ms}, V_{\mathrm{bg}} 40 \mathrm{~V}$ ) were applied, and the pulse interval $(\Delta t)$ ranged from 0.7 to $3.5 \mathrm{~s}$. The PPF-like behavior was remarkably observed, in Fig. $3 \mathrm{e}$, and the PPF ratio $\left(\Delta W_{2}, \Delta W_{n}\right.$ represents the percentage of the postsynaptic responses triggered by the $n^{\text {th }}$ to $1^{\text {st }}$ input spikes, where $n$ is greater than 1) grew to saturation as $\Delta t$ increased. Moreover, a similar phenomenon occurred when ten sequential presynaptic spikes impinged on the artificial InSe synapse, at the same light power density, spike duration, and $V_{\mathrm{bg}}$ condition. As the frequency increased, the PSC gain $\left(\Delta W_{10}\right)$ faded in Fig. 3f. Such observations differ from those reported in most studies on PPF, but have been validated in the human brain ${ }^{39,53}$, and attributed to the release of the captured carriers at the InSe/ $\mathrm{SiO}_{2}$ interface under the negative $V_{\mathrm{bg}}$. Therefore, the increased pulse interval facilitated the charges in the InSe channel, which resulted in higher values for the PSC, the PPF ratio, and the PSC gain. The results corresponded to an improvement of the medicine-acting effect with a relative ascent in the interval following the administration of the adequate dose of a 
a

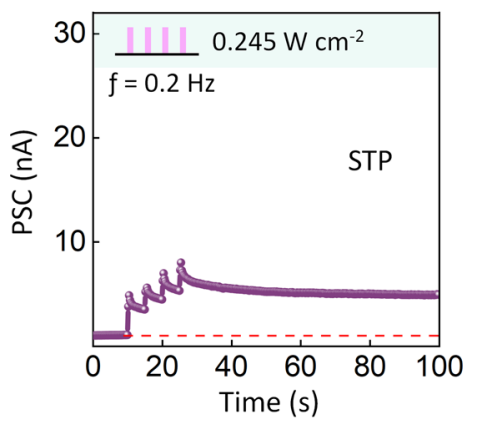

b

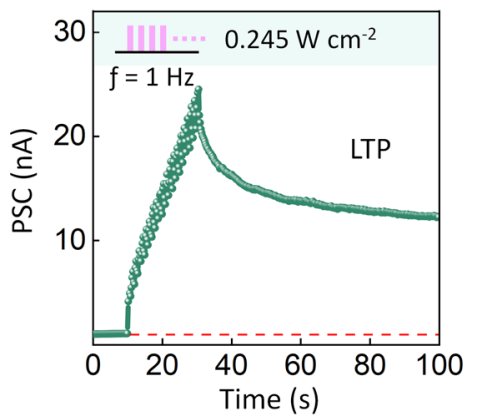

C
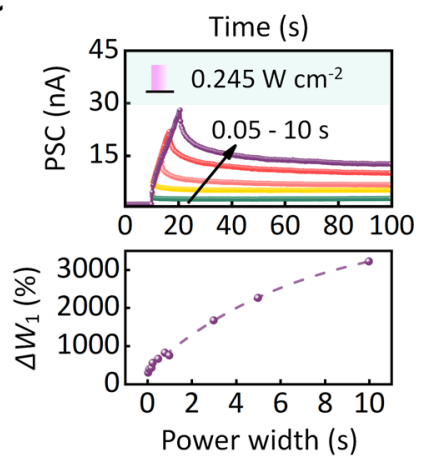

d
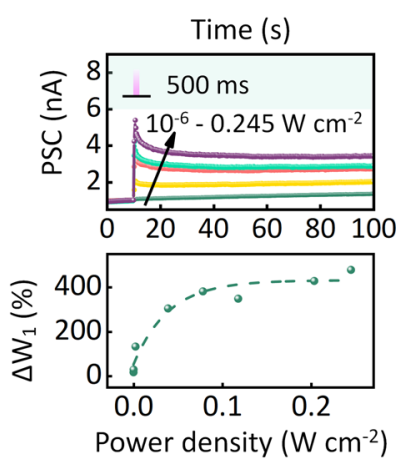

e
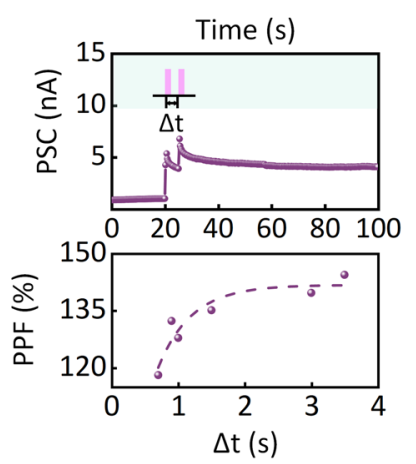

f

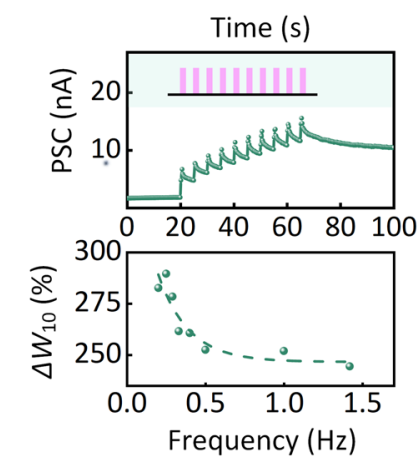

Fig. 3 Synaptic plasticity of the InSe-based device. a Short-term plasticity (STP) and b long-term plasticity (LTP) behavior of the InSe optoelectronic synapse illuminated by a $405-\mathrm{nm}$ laser with a pulse width of $500 \mathrm{~ms}$, a power density of $0.245 \mathrm{~W} \mathrm{~cm}{ }^{-2}$, and frequency of $0.2 \mathrm{~Hz}$ (STP) and $1 \mathrm{~Hz}$ (LTP). The amplitudes and weight variations $\left(\Delta W_{1}\right)$ of the postsynaptic current (PSC) under different c presynaptic pulse widths increasing from 0.05 to $10 \mathrm{~s}$ and d illumination intensity ranging from $10^{-6}$ to $0.245 \mathrm{~W} \mathrm{~cm}^{-2}$. e Paired-pulse facilitation (PPF) emulated by two identical presynaptic spikes (power intensity of $0.245 \mathrm{~W} \mathrm{~cm}^{-2}$, exposure duration of $500 \mathrm{~ms}$ ) and PPF ratio as a function of pulse interval ( $\Delta t$ ). f PSC response triggered by a series of ten presynaptic spikes and the weight change of PSC $\left(\Delta W_{10}\right)$ as a function of frequency. Note that all results were done at $V_{\mathrm{bg}}=-40 \mathrm{~V}$ and $V_{\mathrm{ds}}=0.5 \mathrm{~V}$.

medication. Conversely, the frequent use drugs reduced the treatment efficacy.

To systematically investigate the dependency of synaptic activity on the modulation of the gate voltage as well as to realize the medicine-acting effect, we further measured the metaplasticity in the InSe optoelectronic devices by varying $V_{\mathrm{bg}}$ from -80 to 80 V. Combining Fig. $4 a$ and Supplementary Figs. 5-8, it can be appreciated that the amplitude of PSC obviously increased at a negative $V_{\mathrm{bg}}$ and dropped off at a positive $V_{\mathrm{bg}}$ which corresponded to the two reactions (i.e., effective and ineffective drug use) under adequate and ultralow drug doses. These opposite variations caused by the reversed electric field polarities in the PSC are also highlighted in Supplementary Fig. 4. Furthermore, the presynaptic pulses of various frequencies with illumination cycles of $20 \mathrm{~s}$ were applied at each gate voltage conditions $(-80,-40,0,40$, and $80 \mathrm{~V})$. The observations suggest that the presynaptic spike frequency steered the degree of variation in synaptic weight $\left(\Delta W_{n}\right)$. For convenient comparison, we stored the synaptic weight in a $3 \times 3$ matrix and utilized the elements to represent different $V_{\mathrm{bg}}$ values plotted in Fig. $4 \mathrm{~b}$. The results recorded in Fig. $4 \mathrm{c}$ show that when the pulse frequency ranged from 0.2 to $1.25 \mathrm{~Hz}$ in exposure cycles of $20 \mathrm{~s}, \Delta W_{n}$ gradually increased at negative $V_{\mathrm{bg}}$ values, became flat at zero bias and decreased at positive voltages respectively. Apart from varying pulse frequencies, the number of pulses can also be used to modify the updating of synaptic weight as shown in Fig. $4 \mathrm{~d}$ and Supplementary Fig. 9. The InSe-based synapse was triggered by $\sim 100$ presynaptic spikes with the pulse frequency of $1 \mathrm{~Hz}$ under different gate voltages. We recorded the PSCs and calculated the corresponding $\Delta W_{n}$ value, which indicated that the synaptic weight enhanced and then stabilized at $V_{\mathrm{bg}}$ of -80 and $-40 \mathrm{~V}$ with the incremental pulse number, in close accordance with effective treatment by administering the drug at an adequate dosage exhibited in Fig. $2 \mathrm{~b}$. However, when a positive bias (i.e., $V_{\mathrm{bg}}$ of 40,60 , and $80 \mathrm{~V}$ ) was applied to the gate electrode, the $\Delta W_{n}$ followed a reverse tendency, which was similar to the ineffective drug efficacy at a low dosage. Besides, as the $V_{\mathrm{bg}}$ increased, the decay of $\Delta W_{n}$ became more prominent. Such a phenomenon profits from the release of the trapped charges at negative $V_{\mathrm{bg}}$ values, and the capture of the charges at the interface between the InSe and $\mathrm{SiO}_{2}$ at positive $V_{\mathrm{bg}}$ values. At $V_{\mathrm{bg}}=0 \mathrm{~V}$, few carriers were trapped or detrapped, and the synaptic weight barely changed, which was analogous to stable treatment. Accordingly, the gate voltage, serving as the dosage of the drug, can dynamically imitate the effective/stable/ineffective drug responses in a patient (in Fig. 2b), meanwhile a higher drug stimulus frequency and a greater number of spikes causes a deeper degree of medicine-acting response.

\section{Carrier fluctuations mechanism of InSe-based synapse}

For a more intuitive comprehension of the mechanism underlying the carrier fluctuations in InSe optoelectronics, we carried out systematic dynamic characteristic measurements under various conditions. Figure $5 \mathrm{a}$ indicates the power spectral map of the current fluctuations $\left(S_{1}\right)$ at various $V_{\mathrm{bg}}$ as a function of frequency. As the measurement frequency increased from 10 to $10^{4} \mathrm{~Hz}$ and gate voltage came to the more positive value, $S_{\text {I }}$ was gradually aggrandized. Besides, the variations of $S_{I}$ with increasing frequency at $V_{\mathrm{bg}}=-40,0,40 \mathrm{~V}$ and with increasing $V_{\mathrm{bg}}$ at a fixed frequency of $20 \mathrm{~Hz}$ were highlighted in the inset at the bottom 
a
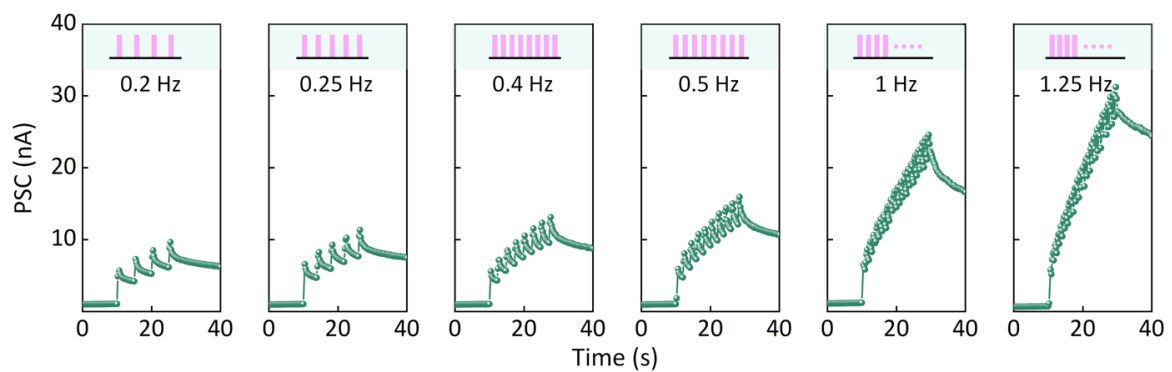
$\Delta W_{n}(\%)$

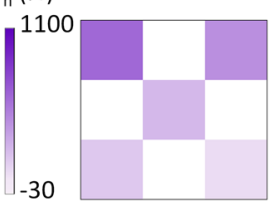

$0.2 \mathrm{~Hz}$

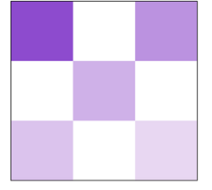

$0.25 \mathrm{~Hz}$

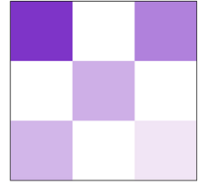

$0.4 \mathrm{~Hz}$

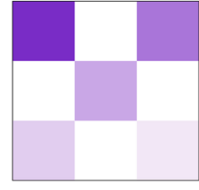

$0.5 \mathrm{~Hz}$

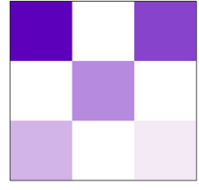

$1 \mathrm{~Hz}$

d
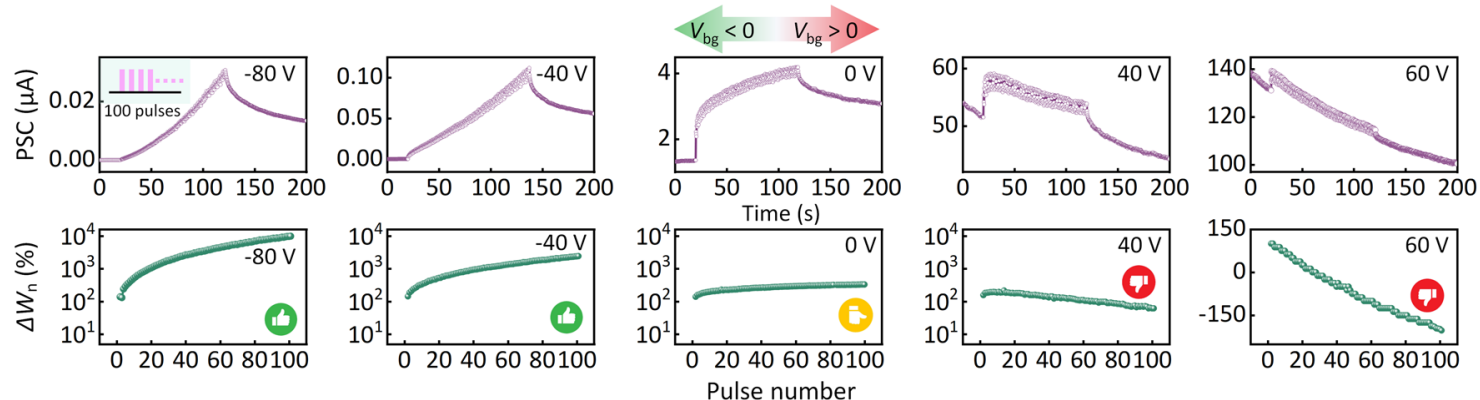

Fig. 4 Metaplasticity in the InSe synaptic devices under various frequencies and numbers of presynaptic spikes. a PSC generated by presynaptic illumination with a $405-\mathrm{nm}$ laser $\left(0.245 \mathrm{~W} \mathrm{~cm}^{-2}, 500 \mathrm{~ms}\right)$ with diverse frequencies of $0.2,0.25,0.4,0.5,1$, and $1.25 \mathrm{~Hz}$, at $V_{\mathrm{bg}}=$ -40 V. b Diagram showing different gate voltages represented by elements of the matrix. c Maps for weight change of the PSC $\left(\Delta W_{n}\right)$ under various $V_{\mathrm{bg}}$ at the aforementioned frequencies. d PSC responses triggered by a train of 100 presynaptic spikes and the corresponding $\Delta W_{n}$ under different $V_{\mathrm{bg}}$ ranging from -80 to $60 \mathrm{~V}$.

and right of Fig. $5 a$, respectively. Among which, the $S_{1}$ curves under each gate voltage were consistent with the representative ideal $1 / f$ signal, revealing that carrier capture or scattering was uniformly distributed both in terms of space and energy. The $S_{1}$ was further normalized by the square of the $I_{\mathrm{ds}}$ at various $V_{\mathrm{ds}}$ values, as shown in Fig. 5b. What is noteworthy is that the normalized $S_{1}$ independent of $V_{\mathrm{ds}}$ suggested that the LFN originated from the $\mathrm{InSe} / \mathrm{SiO}_{2}$ interface, rather than from the contact barriers between the source/drain electrodes and the InSe channel $^{34}$. This corroborated by the highly linear output characteristics in Fig. 1e, which exhibits good Ohmic contact behavior. Moreover, it is remarkable that effective trap density $\left(N_{\text {it }}\right)$ at a fixed frequency can be characterized using the formula $N_{\text {it }} \propto \frac{s_{l}}{g_{m}^{2}}$, where $g_{\mathrm{m}}$ is the transconductance. And the formula is determined by the carrier number (CNF) model based on carrier capturing and releasing events near the dielectric/semiconductor interface. Hence, the $N_{\text {it }}$ depends heavily on the gate electric field owing to gate bias-modulated $g_{\mathrm{m}}{ }^{42,43}$. Combining with Fig. $5 \mathrm{a}$ and Supplementary Fig. 3, we can conclude that the gate voltagemodulated metaplasticity and the $\Delta W_{n}$ characteristics with opposite trends are derived from carrier capturing/releasing events, as well as from the $N_{\text {it }}$ variations between the InSe semiconductor and the $\mathrm{SiO}_{2}$ substrate. We further described the dynamic floating process, and used energy-band diagrams to elucidate the functional mechanism underlying the InSe synaptic optoelectronics in Fig. $5 c, d$, respectively. When we applied a positive $V_{\mathrm{bg}}$ to the InSe optoelectronics, the energy band of the InSe surface bent downward, which caused more carriers to travel from the channel to the $\operatorname{InSe} / \mathrm{SiO}_{2}$ interface where they were captured by the interface trap states. It is a remarkable fact that the traps multiplied sharply with more positive $V_{\mathrm{bg}}$ on account of a higher position of the Fermi level in the InSe. Furthermore, with increasing amounts of light stimulation, a greater number of the carriers were trapped, shielding the effect of the effective gate electric field, and then leading to a more obvious reduction of $I_{\mathrm{ds}}$ in the InSe channel, as exhibited in Supplementary Fig. 10. On the contrary, the captured carriers were released from the $\mathrm{SiO}_{2}$ surface with a negative $V_{\mathrm{bg}}$ implementing an increase synaptic weight change $\left(\Delta W_{n}\right)$, which profited from an ever-larger PSC triggered by a series of light stimuli training. Above this all, our InSe-based optoelectronics with gate-modulated photoresponses could successfully imitate effective/stable/ineffective medicine-acting metaplasticity, as shown in Fig. $2 b$.

To summarize, we demonstrated a carrier-capture-assisted InSe optoelectronic device and successfully imitated medicine-acting metaplasticity with aid of the strong dependence on polarity of the applied electric fields. In our work, the InSe transistor achieved excellent electronical and photoelectrical properties due to the high mobility, optical sensitivity, and low power consumption. In addition, the STP, LTP, and PPF behaviors tuned by the photic stimulus emulated the drug-related synaptic plasticity of the human brain. Owing to the trap capture/release characteristics of the interface between the InSe semiconductor and the $\mathrm{SiO}_{2}$ dielectric layer, the highly gate-modulated photoresponse supported the emulation of effective/stable/ineffective features triggered by repeated drug stimuli with adequate/low/ultralow 
a
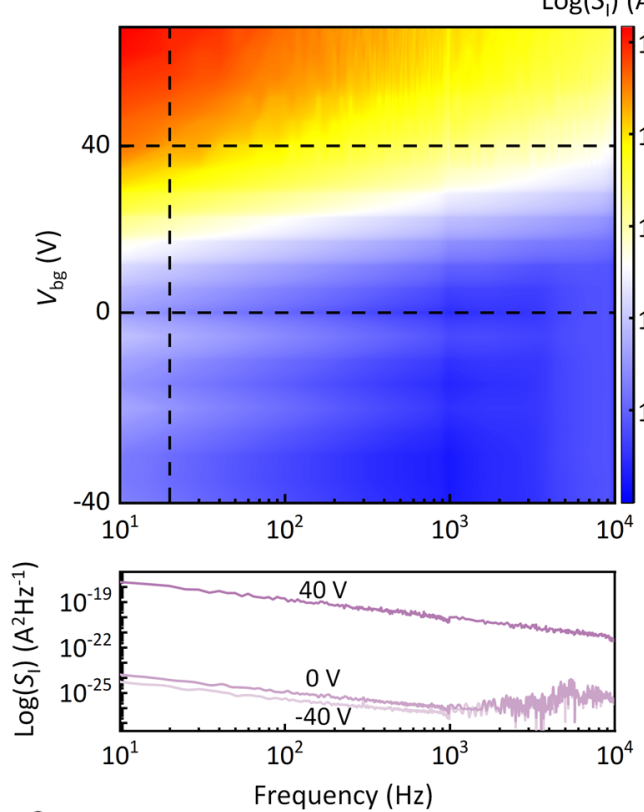

C

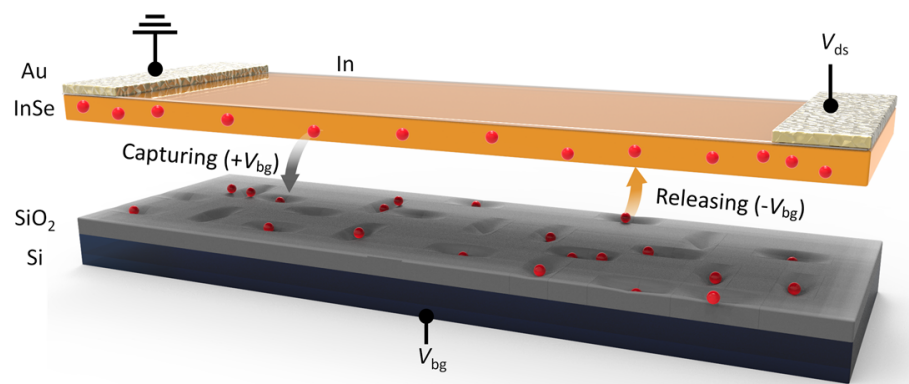

b

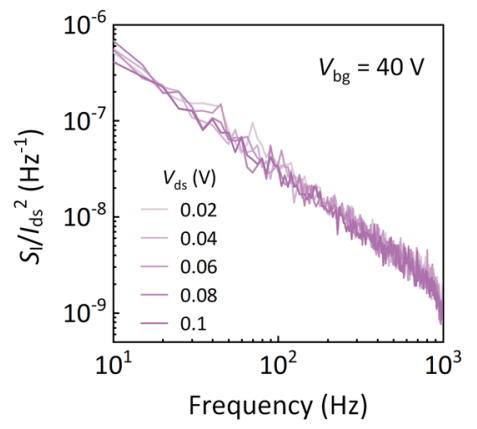

d
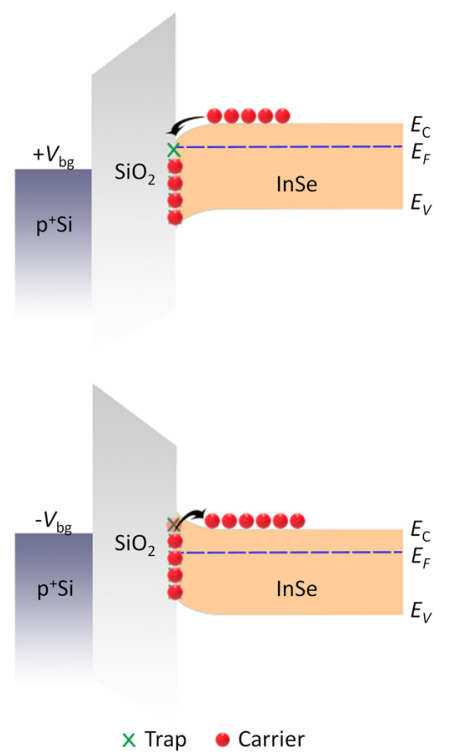

Fig. 5 Mechanism of carrier fluctuations and energy-band diagrams. a Power spectral map of the current fluctuations $\left(S_{1}\right)$ at different $V_{b g}$ as a function of frequency, the curves of $S_{1}$ versus frequency at $V_{\mathrm{bg}}=-40,0$, and $40 \mathrm{~V}$ are listed at the bottom and the $S_{\mathrm{I}}-V_{\mathrm{bg}}$ curve at $20 \mathrm{~Hz}$ is plotted on the right. $\mathbf{b}$ Normalized $S_{\mathrm{I}}$ as a function of frequency at diverse $V_{\mathrm{ds}}$ and $V_{\mathrm{bg}}=40 \mathrm{~V}$. c Schematic of the trapping/detrapping events at the interface between the InSe semiconductor and the $\mathrm{SiO}_{2}$ layer. $\mathbf{d}$ Energy-band diagrams of the carrier capture and release process. Note the corresponding band diagrams of the InSe-based synaptic device under light illumination are shown in the Supplementary Information.

dosages at the device level, corresponding to ascending/flattening/descending $\Delta W_{n}$ indexes under negative/zero/positive bias, respectively. In addition, the LFN measurements further illustrated that the tunable metaplasticity and $\Delta W_{n}$ variation trend originated from carrier capturing/releasing. Therefore, the proposed synaptic optoelectronics could pave the way for a connection between electronics and neurology.

\section{METHODS}

\section{Device fabrication}

Atomically layered InSe flakes were mechanically exfoliated from the bulk crystal using the conventional Scotch tape method and transferred to a $\mathrm{p}^{+}$ silicon substrate capped with a $300 \mathrm{~nm}$ oxide dielectric $\mathrm{SiO}_{2}$ layer. The $\mathrm{p}^{+}$ silicon substrate served as a back-gate. Subsequently, an In layer with a thickness of $30 \mathrm{~nm}$ was intentionally deposited on the top surface of a multilayered InSe film as a doping/packed layer. It should be emphasized that the surface morphology of the deposited In doping/packed layer shows inconsecutive film, displaying ignorable contributions to conducting paths for charge transport. Drain-source electrodes (Au) were fabricated by thermal evaporation using a shadow mask. The detail of the device fabrication was given elsewhere ${ }^{34}$.

\section{Characterizations}

The layer thickness was determined through AFM system (Dimension Icon, Bruker), and the morphology of the InSe FET was identified via optical microscopy (Olympus, BX51M). Raman spectra were measured by Raman microscope (Jobin Yvon LabRAM HR Evolution, Horiba) with an excitation laser of $532 \mathrm{~nm}$.

\section{Electrical properties}

The electrical characterizations of optoelectronic devices based on multilayer InSe were performed by using a semiconductor parameter analyzer (Keithley, 4200-SCS) under a high vacuum condition to avoid channel oxidation ${ }^{6}$. Note that all the electrical behaviors of the InSe devices were determined in darkness to reduce fluctuations due to the environmental perturbations. The photoresponses of the devices were carried out using an adjustable laser driver system (Thorlabs, ITC4001). All the measurements were obtained at room temperature.

\section{DATA AVAILABILITY}

Data are available on request from the authors.

Received: 14 December 2020; Accepted: 13 May 2021; Published online: 10 June 2021 


\section{REFERENCES}

1. John, R. A. et al. Flexible ionic-electronic hybrid oxide synaptic TFTs with programmable dynamic plasticity for brain-inspired neuromorphic computing. Small 13, 1701193 (2017).

2. Seo, S. et al. Artificial van der Waals hybrid synapse and its application to acoustic pattern recognition. Nat. Commun. 11, 3936 (2020).

3. $\mathrm{Li}$, J. et al. Symmetric ultrafast writing and erasing speeds in quasi-nonvolatile memory via van der Waals heterostructures. Adv. Mater. 31, 1808035 (2019).

4. Mukherjee, B. et al. Laser-assisted multilevel non-volatile memory device based on 2D van-der-Waals few-layer-ReS2/h-BN/graphene heterostructures. Adv. Funct. Mater. 30, 2001688 (2020).

5. Hong, S. et al. Complementary type ferroelectric memory transistor circuits with P- and N-channel MoTe 2. Adv. Electron. Mater. 6, 2000479 (2020).

6. Yang, F. S. et al. Oxidation-boosted charge trapping in ultra-sensitive van der Waals materials for artificial synaptic features. Nat. Commun. 11, 2972 (2020).

7. Clement, N., Nishiguchi, K., Fujiwara, A. \& Vuillaume, D. One-by-one trap activation in silicon nanowire transistors. Nat. Commun. 1, 92 (2020).

8. Pak, J. et al. Trapped charge modulation at the $\mathrm{MoS}_{2} / \mathrm{SiO}_{2}$ interface by a lateral electric field in $\mathrm{MoS}_{2}$ field-effect transistors. Nano Futures 3, 011002 (2019).

9. Guo, Y. et al. Charge trapping at the $\mathrm{MoS}_{2}-\mathrm{SiO}_{2}$ interface and its effects on the characteristics of $\mathrm{MoS}_{2}$ metal-oxide-semiconductor field effect transistors. Appl. Phys. Lett. 106, 103109 (2015).

10. Li, Q., Zhou, Q., Shi, L., Chen, Q. \& Wang, J. Recent advances in oxidation and degradation mechanisms of ultrathin $2 \mathrm{D}$ materials under ambient conditions and their passivation strategies. J. Mater. Chem. A 7, 4291-4312 (2019).

11. Kang, K. et al. Graphene-assisted antioxidation of tungsten disulfide monolayers: substrate and electric-field effect. Adv. Mater. 29, 1603898 (2017).

12. Yoon, J. H. et al. An artificial nociceptor based on a diffusive memristor. Nat. Commun. 9, 417 (2018).

13. Feng, G. et al. A sub-10 nm vertical organic/inorganic hybrid transistor for painperceptual and sensitization-regulated nociceptor emulation. Adv. Mater. 32, 1906171 (2020).

14. Bi, G. Q. \& Poo, M. M. Synaptic modifications in cultured hippocampal neurons: dependence on spike timing, synaptic strength, and postsynaptic cell type. J. Neurosci. 18, 10464-10472 (1998).

15. Lauri, S. E. et al. Presynaptic mechanisms involved in the expression of STP and UP at CA1 synapses in the hippocampus. Neuropharmacology 52, 1-11 (2007).

16. Wang, Y. et al. Photonic synapses based on inorganic perovskite quantum dots for neuromorphic computing. Adv. Mater. 30, 1802883 (2018).

17. Di Chiara, G. Drug addiction as dopamine-dependent associative learning disorder. Eur. J. Pharmacol. 375, 13-30 (1999).

18. Ohno, T. et al. Short-term plasticity and long-term potentiation mimicked in single inorganic synapses. Nat. Mater. 10, 591-595 (2011).

19. Sangwan, V. K. et al. Multi-terminal memtransistors from polycrystalline monolayer molybdenum disulfide. Nature 554, 500-504 (2018).

20. Costenla, A. R., Cunha, R. A. \& de Mendonca, A. Caffeine, adenosine receptors, and synaptic plasticity. J. Alzheimer's Dis. 20, S25-S34 (2010).

21. Fagen, Z. M., Mansvelder, H. D., Keath, J. R. \& McGehee, D. S. Short- and long-term modulation of synaptic inputs to brain reward areas by nicotine. Ann. N. Y. Acad. Sci. 1003, 185-195 (2003).

22. Kvarta, M. D., Harris-Warrick, R. M. \& Johnson, B. R. Neuromodulator-evoked synaptic metaplasticity within a central pattern generator network. J. Neurophysiol. 108, 2846-2856 (2012).

23. Martins, D., Mehta, M. A. \& Prata, D. The "highs and lows" of the human brain on dopaminergics: Evidence from neuropharmacology. Neurosc. Biobehav. Rev. 80, 351-371 (2017).

24. Wise, R. A. Drug-activation of brain reward pathways. Drug Alcohol Depend. 51, 13-22 (1998).

25. Shim, H. et al. Stretchable elastic synaptic transistors for neurologically integrated soft engineering systems. Sci. Adv. 5, eaax4961 (2019).

26. Wang, K. et al. Light-stimulated synaptic transistors fabricated by a facile solution process based on inorganic perovskite quantum dots and organic semiconductors. Small 15, 1900010 (2019).

27. Kwon, S. M. et al. Environment-adaptable artificial visual perception behaviors using a light-adjustable optoelectronic neuromorphic device array. Adv. Mater. 31, 1906433 (2019).

28. Chen, Y. et al. Piezotronic graphene artificial sensory synapse. Adv. Funct. Mater. 29, 1900959 (2019).

29. Sun, Y. et al. Photoelectric synaptic plasticity realized by 2D perovskite. Adv. Funct. Mater. 29, 1902538 (2019).

30. Wang, L. et al. Artificial synapses based on multiterminal memtransistors for neuromorphic application. Adv. Funct. Mater. 29, 1901106 (2019).

31. Wang, S. et al. A MoS 2 /PTCDA hybrid heterojunction synapse with efficient photoelectric dual modulation and versatility. Adv. Mater. 31, 1806227 (2019).
32. Arnold, A. J. et al. Mimicking neurotransmitter release in chemical synapses via hysteresis engineering in $\mathrm{MoS}_{2}$ transistors. ACS Nano 11, 3110 (2017).

33. Ahmed, T. et al. Optically stimulated artificial synapse based on layered black phosphorus. Small 15, 1900966 (2019).

34. Li, M. et al. High mobilities in layered inse transistors with indium-encapsulationinduced surface charge doping. Adv. Mater. 30, 1803690 (2018).

35. Chen, F. et al. Lattice vibration characteristics in layered InSe films and the electronic behavior of field-effect transistors. Nanotechnology 31, 335702 (2020).

36. Tamalampudi, S. R. et al. High performance and bendable few-layered InSe photodetectors with broad spectral response. Nano Lett. 14, 2800-2806 (2014).

37. Chen, L. et al. Ultrasensitive and robust two-dimensional indium selenide flexible electronics and sensors for human motion detection. Nano Energy 76, 105020 (2020).

38. Chen, L. et al. Ultrasensitive flexible strain sensor based on two-dimensional InSe for human motion surveillance. In: 2019 IEEE International Electron Devices Meeting (IEDM), 26.26.1-26.26.4 (IEEE, 2019).

39. Lee, K.-C. et al. Inverse paired-pulse facilitation in neuroplasticity based on interface-boosted charge trapping layered electronics. Nano Energy 77, 105258 (2020).

40. Choi, M. S. et al. Controlled charge trapping by molybdenum disulphide and graphene in ultrathin heterostructured memory devices. Nat. Commun. 4, 1624 (2013).

41. Lee, J. et al. Monolayer optical memory cells based on artificial trap-mediated charge storage and release. Nat. Commun. 8, 14734 (2017).

42. Lin, Y. F. et al. Origin of noise in layered $\mathrm{MoTe}_{2}$ transistors and its possible use for environmental sensors. Adv. Mater. 27, 6612-6619 (2015).

43. Lee, J. W. et al. Sidewall crystalline orientation effect of post-treatments for a replacement metal gate bulk fin field effect transistor. ACS Appl. Mater. Inter. 5, 8865-8868 (2013).

44. Mudd, G. W. et al. Tuning the bandgap of exfoliated InSe nanosheets by quantum confinement. Adv. Mater. 25, 5714-5718 (2013).

45. Lei, S. D. et al. Evolution of the electronic band structure and efficient photodetection in atomic layers of InSe. ACS Nano 8, 1263-1272 (2014).

46. Yin, L. et al. Ultrafast and ultrasensitive phototransistors based on few-layered HfSe2. Appl. Phys. Lett. 109, 213105 (2016).

47. Liao, F. et al. A dual-gate $\mathrm{MoS}_{2}$ photodetector based on interface coupling effect. Small 16, 1904369 (2020).

48. Koppens, F. H. et al. Photodetectors based on graphene, other two-dimensional materials and hybrid systems. Nat. Nanotechnol. 9, 780-793 (2014).

49. Zhao, Q. et al. The role of traps in the photocurrent generation mechanism in thin InSe photodetectors. Mater. Horiz. 7, 252-262 (2020).

50. Wu, Y. C. et al. Extrinsic origin of persistent photoconductivity in monolayer $\mathrm{MoS}_{2}$ field effect transistors. Sci. Rep. 5, 11472 (2015).

51. Peimyoo, N. et al. Laser-writable high-k dielectric for van der Waals nanoelectronics. Sci. Adv. 5, eaau0906 (2015).

52. Duan, N. et al. An electro-photo-sensitive synaptic transistor for edge neuromorphic visual systems. Nanoscale 11, 17590-17599 (2019).

53. Wang, J. H. \& Kelly, P. T. Attenuation of paired-pulse facilitation associated with synaptic potentiation mediated by postsynaptic mechanisms. J. Neurophysiol. 78, 2707-2716 (1997).

\section{ACKNOWLEDGEMENTS}

This work was financially supported by the National Key Research and Development Program of China (Grant No. 2016YFB0501604), the National Natural Science Foundation of China (Grant Nos 61774061 and 61504043), the NSAF Foundation of China (Grant No. U1830130), the Natural Science Foundation of Shanghai (Grant No. 19ZR1473400), and the Taiwan Ministry of Science and Technology (Grant Nos MOST 109-2221-E-005-011- and 109-2112-M-005-013-MY3). The authors wish to acknowledge Dr Wei-Ju Lee, attending physician of Neurological Institute, Taichung Veterans General Hospital, Taiwan, for his generous help in interpreting the significance of the results of this study.

\section{AUTHOR CONTRIBUTIONS}

W.L. and Y.-F.L. conceived and designed the experiments. M.L. contributed to the device fabrication and LFN measurements. C.G. and Q.N. fabricated the device and conducted the electrical and optical measurements and data analysis. F.-S.Y. and X.W. contributed to the Schematic sketch and AFM measurement. K.-C.L. and C.-Y.L. helped with the data analysis. The paper was written by Q.N. and C.G. with contributions from all the co-authors. W.L. and Y.-F.L. supervised the research. C.-H.H. provided InSe materials. C.-H.L. and S.-P.L. helped design the experiments. Z.H. and J. C. provided the some experimental equipments. All the authors discussed the results and commented on the manuscript. 


\section{COMPETING INTERESTS}

The authors declare no competing interests.

\section{ADDITIONAL INFORMATION}

Supplementary information The online version contains supplementary material available at https://doi.org/10.1038/s41699-021-00241-0.

Correspondence and requests for materials should be addressed to M.L., Y.-F.L. or W.L.

Reprints and permission information is available at http://www.nature.com/reprints

Publisher's note Springer Nature remains neutral with regard to jurisdictional claims in published maps and institutional affiliations.
Open Access This article is licensed under a Creative Commons Attribution 4.0 International License, which permits use, sharing, adaptation, distribution and reproduction in any medium or format, as long as you give appropriate credit to the original author(s) and the source, provide a link to the Creative Commons license, and indicate if changes were made. The images or other third party material in this article are included in the article's Creative Commons license, unless indicated otherwise in a credit line to the material. If material is not included in the article's Creative Commons license and your intended use is not permitted by statutory regulation or exceeds the permitted use, you will need to obtain permission directly from the copyright holder. To view a copy of this license, visit http://creativecommons. org/licenses/by/4.0/.

(c) The Author(s) 2021 